\title{
Research news
}

\section{Identifying new T-cell regulators \\ Diane Martindale}

\author{
Published: 22 September 2003
}

Journal of Biology 2003, 2:17

The electronic version of this article is the complete one and can be found online at http://jbiol.com/content/2/3/17

(c) 2003 BioMed Central Ltd

\section{Functional genomic screening for new components of the signaling pathway that is triggered when $\mathbf{T}$ cells are activated has identified novel signaling molecules that are potential drug targets.}

Signaling by the T-cell receptor (TCR) is a well-trodden cellular pathway, and many of the proteins involved have been identified and their function determined in the past two decades. But therapeutic drugs that target these signaling molecules and alter the response of $\mathrm{T}$ cells to activation by antigens remain scarce. Uncovering novel proteins on the signaling pathway downstream of activation of the TCR would therefore not only help us to understand immune responses better but also aid in the development of new, more effective drugs to treat autoimmune diseases such as lupus and rheumatoid arthritis.

But unraveling this complex pathway and identifying all the players is a long and painstaking task. In this issue of Journal of Biology [1], Peter Chu, Jorge Pardo, Donald Payan, Charlene Liao and colleagues describe a large-scale functional genetic screen that is in theory capable of picking up most, if not all, of the molecules of the TCR signaling pathway. Their work will benefit researchers in immunology and other fields who are eager to identify all the players in a particular signaling pathway (see 'The bottom line' box for a summary of the work).

\section{Building on the past}

The bulk of known TCR signaling proteins (see the 'Background' box) have been uncovered by classical genetic and biochemical methods, such as immunoprecipitation and chemically induced DNA mutagenesis. For the TCR and other cellular signaling pathways, "this is how the chains got built," says Donald Payan of Rigel Pharmaceuticals

\section{The bottom line}

- A genome-wide functional screening protocol has been developed that has the potential to identify all the signaling molecules involved in the pathway downstream of the T-cell receptor, looking for new drug targets.

- A retroviral fragmented cDNA library, made from lymphocyte mRNA, allowed the isolation and recovery of sequences encoding dominant effectors of TCR signaling, as measured by failure of cells to upregulate the cell-surface marker CD69 in response to an activation trigger.

- Many of the 40 or so proteins identified in the screen were already known to play roles in TCR signaling, while several others were known proteins but had not been reported to have a role in TCR signaling. More than a dozen novel proteins were found; some were studied further, to validate their function in a physiological setting.

- Although only one specific protein was identified as a potential novel T-cell drug target, the technique provides a highly efficient strategy to map entire signaling pathways and is applicable to a wide spectrum of cell types. 


\section{Background}

- Signaling by the T-cell receptor (TCR) is triggered when the TCR recognizes antigens (usually from foreign proteins) presented by the major histocompatibility (MHC) proteins of antigenpresenting cells. When the TCR is activated, it triggers signaling through a pathway from the cell surface through to transcription factors in the nucleus.

- Dominant effectors are mutated or truncated gene sequences that encode a truncated version of the protein of interest that has the ability to block the function of its wild-type counterpart or to represent an activated form of its wild-type counterpart.

- The human T-cell leukemia line Jurkat can be successfully infected with retroviruses because it is an immortalized cell and is capable of cell division. The retroviruses can be used to carry cDNAs from a library into the Jurkat cells, where they are then expressed.

- Fluorescence-activated cell sorting (FACS) is a way of rapidly separating cells in a suspension on the basis of their size and/or fluorescence. It is ideally suited to recovering cells with rare phenotypic traits from large cell populations.

in South San Francisco, where the work was carried out. "It's a time-honored and extremely successful method."

In the mid-1990s, researchers turned to retrovirus-based gene transfer as a way to introduce genes into mammalian cells [2]. This powerful technology contributed to the cloning of T-cell antigens, tumor antigens, various receptors, signaling molecules and transcription factors (reviewed in [2]). In many of the cases, however, retroviral cDNA libraries were used for simple expression cloning, rather than for functional cloning, omitting the element of functional screening from the assay. And until now, no one had attempted to use this type of genetic screening tool to functionally map out an entire signaling pathway, from cell membrane to nucleus and everything in between.

\section{Large-scale screening}

The large-scale functional screening approach developed by Chu et al. [1] used a library of retroviral fragmented cDNAs from lymphoid tissues to isolate sequences encoding dominant effectors - fragmented proteins that disrupt the TCR signaling pathway. Expression of some of the sequences inserted in the library had dominant effects over their endogenous counterparts, either by competitively inhibiting the endogenous protein or by being constitutively active. Ultimately, the researchers hoped that this global functional screen would identify new T-cell drug targets.

The first step was to make the cDNA library. To do this, the researchers extracted mRNA from lymphoid organs: the thymus, bone marrow, lymph nodes and spleen. Random primers were used to generate a huge cDNA library containing gene fragments of various sizes that were each individually packaged into the Moloney murine leukemia (retro)virus. One of the keys to this large dominant-effector genetic screen, explains Charlene Liao, now at Genentech in South San Francisco and senior author of the Journal of Biology article, was to use a randomly primed cDNA library since this increases the chance of expressing truncated proteins with potential dominant-negative or constitutively active functions.

Millions of Jurkat cells were infected with the retrovirus library and activated with an anti-TCR antibody that mimics what occurs when $\mathrm{T}$ cells are activated by a natural ligand. To find and recover those rare cells carrying a cDNA insert affecting TCR signaling, the authors chose to screen for the expression of CD69, a cell-surface marker that is associated with T-cell activation. The rationale was to identify cell clones whose CD69 upregulation (a sign of T-cell activation) in response to anti-TCR antibody was repressed following introduction of a sequence from the retroviral cDNA library. Library inserts conferring such repression would then represent immune modulators that function to block TCR signal transduction, and thus, proteins that are part of the normal TCR signaling pathway.

Activated cells were fluorescently labeled with an anti-CD69 marker and those with low CD69 expression selected by fluorescence-activated cell sorting (FACS). After four rounds of reiterative sorting, the group ended up with nearly 3,000 clones of potential interest. To be certain that the phenotype of these clones was due to the expression of the cDNA library rather than to epigenetic changes or insertional mutations, each of the selected clones was further screened to ensure that the phenotype occurred only when expression of the insert was induced (by a derivative of the drug tetracycline). Nearly half of the original clones were selected in this way for further analysis. The cDNA insert from each of these clones was recovered and its sequence determined. When the sequencing was done, the group had uncovered nearly 40 different 


\section{Behind the scenes}

Journal of Biology asked Donald Payan and Charlene Liao about how and why the group decided on a large functional-genomic screen for T-cell signaling molecules.

\section{What motivated you to develop this large-scale genetic screening method and to test it in $\mathrm{T}$ cells?}

The motivation for the screen was to see if we could find novel proteins that might be good drug-target candidates for immune regulation and transplant rejection. This effort did in fact result in several new potential drug targets being identified.

\section{How long did it take your group to do the experiments and what were the steps that ensured success?}

This was a multidisciplinary effort combining the expertise of our virology, immunology, high-throughput and molecular biology groups. From start to finish it took about a year and a half to construct the cell lines, make the libraries, do the genetic screens, reconfirm all the hits, and do follow-on confirmatory immunology experiments. As in most large-scale biology experimentation, good teamwork and the patient development of bulletproof reagents and sensitive, reproducible assays is what ensured success.

\section{What was your initial reaction to the results and how were they received by others?}

The responses to the data were very positive, in that we got many known targets that were previously identified as playing a role in the pathway we had targeted. The fact that we found novel targets, despite working in a pathway that had been extensively mined by others, reconfirmed everyone's belief that functional genomics is a very powerful tool for finding new drug targets. Our approach has yielded many new potential drug targets across numerous pathways that are critical to disease processes.

\section{What are the next steps?}

The next step with this technique is to apply it to novel therapeutic areas, and in addition to take the targets we have identified and do further biological validation so we can select which ones will be taken forward into drug screening.

signaling molecules involved in the TCR pathway.

\section{Validating new components}

"At the end of the day, you have a list of genes that all play a role in T-cell activation," says Payan. "The beauty of this approach is that instead of having to sift through the entire genome, you're sifting through the genome in a functional way. It allows you to link a protein with a very specific cellular activity. This is one of the hardest things facing scientists right now in the post-genome era. We've got all these genes, now what do we do? How do we assign function?"

As expected, many of the proteins in the list were already known to be TCR regulators, such as the protein kinases
Lck, ZAP70, Syk and PLC $\gamma 1$. "What's interesting is that the ones we identified were new truncation mutants," says Liao. "Now, we can tell other researchers who have been working in the field for 10 years how to make new dominant-negative mutants or constitutively active mutants of their favorite proteins."

In addition, several other genes, whose sequences had been reported previously but whose involvement in TCR signaling was not documented, were also found in the screen. These included the G-protein-coupled receptor EDG1, which is involved in endothelial cell differentiation; PAK2, a serine/threonine kinase involved in cytoskeletal reorganization and nuclear signaling; and the adaptor molecule Grb7, which mediates the coupling of various cell-surface receptors to downstream signaling pathways. In addition, the authors report more than a dozen novel molecules, including one that they named TRAC-1.

Chu et al. [1] also confirmed the presence of the transcripts encoding these proteins in lymphoid organs using northern blots. Further testing of lymphocyte subsets with semi-quantitative PCR revealed that these same genes were also expressed specifically in $\mathrm{T}$ and $\mathrm{B}$ cells, as well as in peripheral blood mononuclear cells. In a final test to validate the approach, Chu et al. also showed that these genes are indeed relevant to the functioning of primary T lymphocytes.

"This is a Herculean effort: we had millions of cells, thousands of clones, and hundreds of sequences," says Payan. "Many of the proteins we found turned out to be known players in $\mathrm{T}$ cell activation but this validated our system. I just wish we'd had this technique in the early ' 80 s - we could have dominated the whole [T-cell] field." Notably, Payan's group unraveled most, if not all, of the TCR signaling proteins they found in a mere 18 months - a task that has taken others, using standard mapping methods, 15 
or more years (see the 'Behind the scenes' box for more of the motivation behind the work).

Not only is this functional genomic technique highly efficient and fast, it is also applicable to a broad spectrum of cells. "Part of the importance and benefit of this work is that it could be performed on a wide range of cell types, for a wide range of functionally important readouts. As long as the readout can be detected in a rare-event detection screen, the mutants could be identified, amplified, and checked against the genome," says Mark Boothby at Vanderbilt University Medical School in Nashville. For example, it could be used to screen for altered signaling in key target cells after insulin treatment, to find novel genes involved in insulin responsiveness as seen in type II diabetes, as well as in neural cells to screen for mutations affecting ion channels, or handling of serotonin or dopaminergic signaling, which occurs in depression and schizophrenia.

"Another great virtue of the approach is that you make no assumptions as to what the target will be," says Philippa Marrack of the Howard Hughes Medical Institute at the National Jewish Medical and Research Center in Denver. Thus, the search is not biased towards previously known signaling molecules.

But before drugs can be churned out, there's a lot more work to be done. "What the technique doesn't tell you is who's dancing with whom, and which is the more dominant partner, or whether the protein is just part of this T-cell pathway or shared with other pathways, and which molecule comes first, second, third in the chain," admits Payan. "As elegant as this experiment is, it's only a starting point." Before the group can design inhibitory drugs, they will also need to determine which part of each protein is linked to its activity.

Another drawback, notes Boothby, is that the approach operates on a scale at which cell lines need to be used, and like most cell lines, the Jurkat $\mathrm{T}$ cells have pre-existing signaling abnormalities. "Still, this is nice work. It is estimated that we may face the task of finding new functions for as much as 40 percent of the predicted open reading frames in the genome. It will take many different approaches, but this method is one that will help accelerate the process of screening for promising prospects among the 12,000-plus novel genes that may be out there in the human and mouse genomes alone."

Although the current report does not pinpoint which novel molecules were found to be suitable drug targets, the TRAC-1 protein, which is homologous to the ubiquitin ligases and has immune-specific expression, is an interesting candidate. Payan assures us that the company is now focusing its efforts on further characterizing a few of the newly identified molecules. The majority of the proteins found were in the cytoplasm, and that poses another challenge because it is enormously difficult to design drugs that are "cell-permeable with reasonable pharmacokinetics and specific for their targets," notes Marrack.

While the new technique described by Liao, Payan and colleagues might not lead immediately to the next wonder-drug, it has already contributed to T-cell biology by adding several potential players - which had not been picked up by other mapping techniques - to the list of TCR signaling molecules. And, in turn, these might be useful in learning how to manipulate the immune system better and perhaps to overcome afflictions such as cancer, infection and autoimmune diseases.

\section{References}

I. Chu P, Pardo J, Zhao H, Li C, Pali E, Shen M, Qu K, Yu S, Huang B, Yu P, et al.: Systematic identification of regulatory proteins critical for T-cell activation. J Biol 2003, 2:2I.

2. Lorens B, Sousa C, Bennett M, Molineaux $S$, Payan D: The use of retroviruses as pharmaceutical tools for target discovery and validation in the field of functional genomics. Curr Opin Biotechnol 2001, I2:613-62I.

Diane Martindale is a science writer based in Toronto, Canada.

E-mail: dmartindale@writescience.ca 